\title{
The Effects of Denervation and Stimulation upon Synaptic Ultrastructure
}

\author{
L. T. RUTLEDGE \\ Department of Physiology, The University of Michigan Medical School, \\ Ann Arbor, Michigan 48109
}

\begin{abstract}
Quantitative studies of synaptic ultrastructure were made in the upper layers of cat cerebral cortex. Tissues were from intact cortex and from long-term (chronic) undercut cortex with or without electrical stimulation. The synaptic effects of chronic electrical stimulation of denervated cortex are most readily understood as growth and remodeling of synaptic elements. Associated with chronic stimulation were increases in: symmetric membrane contacts; areas of round and flat vesicle containing terminals; dendritic shaft contacts; and synaptic contact lengths. Even without stimulation there were indications of synaptic plasticity in denervated cortex; compared with intact cortex, synapses having symmetric membrane contacts showed an increase in bouton area and an increase in synaptic contacts on dendritic shafts. These data are consistent with the observations of others in which axonal terminal growth occurred after deafferentation. But it appears that chronic electrical stimulation in the adult nervous system promotes significantly more plasticity than occurs without stimulation. In a functional sense stimulation in the present experiments produced effective inhibition which did not occur with denervation alone. Thus the plasticity observed with stimulation had both structural and functional components.
\end{abstract}

Electrical stimulation of the central nervous system (CNS) has been widely used in experimental animals studied over long periods of time. Currently long-term (chronic) stimulation of portions of the human nervous system is receiving increasing attention as a therapeutic procedure (e.g., Cooper et al., '76; Dobelle et al., '76; Fields and Adams, '74; Richardson, '76). In some human cases the effects of CNS stimulation are cumulative and this implies significant alterations in neural function. Whether these changes have a basis in humoral, structural, biochemical or other factors is unknown. An important consideration is to define the effects of chronic electrical stimulation at the synaptic level. We have approached this problem by attempting to prevent degeneration of neocortical neuronal elements, following lesions, by electrical stimulation.

Deafferentation and deefferentation of neurons in the neocortex can be accomplished by surgically undercutting the cortex. (These dual effects upon neurons will be referred to here as "denervation.") This procedure results in retrograde degenerative loss of many efferent cortical neurons and chromatolysis of some of those remaining (Cajal, '59; Rutledge, '69; Rutledge et al., '69; Szentágothai, '65). The undercut tissue shows gliosis, dendritic beady swellings and distortions, loss of dendritic branchings, and loss of dendritic spines. Similar effects were found in an irritative epileptic focus where deafferentation was thought to be the causal process of the epileptic activity (Westrum et al., '64). There was also a significant loss of axon collateral branches (Rutledge et al., '69). Some of the degenerative changes could be prevented by chronic electrical stimulation of undercut cortex (Rutledge, '69; Rutledge et al., '69); spine loss was prevented and axon collateral branches were maintained. These results and others (Anderson et al., '75; Chu et al., '71a,b; Duncan et al., '68; Rutledge et al., '67) indicated that electrical stimulation of denervated cortex preserved neuronal chemistry, structure, and function. To determine the possible alterations in synaptic ultrastructure with chronic stimulation several questions 
were posed. Do synaptic membrane specializations and terminal boutons change in size? Are spines preserved by stimulation located in particular layers of the cortex or in particular regions of a given cell? Are types of synapses distributed differently on cells as a result of stimulation, indicating perhaps specific functional changes? Answers to these and related questions would not only help to understand what happens in stimulated denervated cortex but might be important for explaining some of the therapeutic effects of brain stimulation referred to above. A preliminary report of some of the work to be reported here has appeared (Rutledge, '75).

\section{METHODS}

Data were obtained from adult cats, two in each of three groups, designated, Intact Cortex (I), Undercut Cortex (UC), and Undercut Cortex, Stimulated (UCS). The four experimental animals were prepared in a manner similar to that previously described (Anderson et al., '75; Rutledge et al., '67). Briefly, under sterile surgical conditions and approaching through the lateral aspect of the suprasylvian gyrus, one marginal gyrus in each cat was undercut for $16-20 \mathrm{~mm}$ at a depth of about $4 \mathrm{~mm}$. This resulted in gray and some of the central core of white remaining in the slab throughout the width of the undercut gyrus. A major portion of the blood supply to the marginal gyrus is via pial vessels extending laterally from the midline and this remained largely intact. UC cats were left in home cages for 86 and 270 days before they were killed for tissue preparation. In terms of degenerative changes Szentágothai ('65) believes stability has been reached in two months and Gruner et al. ('74) believes this occurs sooner. Hyperexcitability ("supersensitivity") develops even earlier than this (Rutledge, '69; Rutledge et al., '67; Sharpless and Halpern, '62). The synaptic alterations to be described below did not seem to be related to duration of undercut. At the time of undercutting animals destined for chronic stimulation (UCS) received bilateral electrode implants in the center of the undercut, and in the homotopic area contralaterally, and at approximately the border of areas 17 and 18 (Otsuka and Hassler, '62). Stimulating electrodes were sharpened platinum-iridium needles, $3 \mathrm{~mm}$ apart and extending into cortex about $0.5 \mathrm{~mm}$. Anterior and posterior recording electrodes were $1.5-\mathrm{mm}$ diameter platinum-iridium balls inserted into the skull, but not resting on dura mater, and $4 \mathrm{~mm}$ from the nearest stimulating electrode. Electrical stimulation of undercut cortex was started one day after surgery and consisted of one session daily of 20,2 -sec trains, 0.5 msec pulses, $50 \mathrm{~Hz}$, given at one minute intervals. The square pulses were biphasic. Total stimulations were about 400 at $0.6 \mathrm{~mA}, 400$ at $0.8 \mathrm{~mA}$ and 200 at $1.0 \mathrm{~mA}$, all constant current. The electroencephalogram on both sides of the brain was monitored to ensure that chronic stimulation of undercut cortex did not produce abnormal spiking or afterdischarges. Test stimulations were occasionally applied to each side of the brain to determine relative thresholds for abnormal cortical electrical activity. Over a period of 58 days one UCS cat received 1,075 electrical stimulations, all below intensities which could produce abnormal spiking or afterdischarges. The other UCS cat received 1,055 stimulations over 48 days. As determined repeatedly in previous experiments these periods of stimulation are more than adequate to produce the various changes described, i.e., those associated with the absence of hyperexcitability. Obviously the time since undercutting in UC and UCS is not comparable, but as noted above virtually all degeneration occurs in less than two months. Further a compromise had to be reached for UCS since indwelling electrodes tend to produce increasing amounts of gliosis, thickened dura matter, and perhaps increased vascularity, as they are left in place beyond about two months. Time between the last electrical stimulation and animal sacrifice and tissue fixation was two and four days for UCS.

All cortical tissues were prepared for electron microscopy with a slightly modified Colonnier protocol (personal communication). The anesthetized animal was perfused with a controlled pressure head, beginning at 100 $\mathrm{mm} \mathrm{Hg}$. Before starting the flow of the glutaraldehyde-paraformaldehyde solution about 1 liter of cold normal saline was perfused through the animal's upper body. Following perfusion blocks of marginal gyri $5 \times 5 \times 10$ $\mathrm{mm}$ long were removed, placed in the fixative and kept in a refrigerator overnight. Small blocks were then cut through the cortex with the long orientation surface-to-depth. Blocks from UCS animals were cut from between the electrode locations, and no closer than $1 \mathrm{~mm}$ to them. Comparable areas of marginal gyri in the other animals were sampled. The blocks were then fixed in an osmic acid solution, de- 
hydrated and embedded in Araldite or Araldite-Epon. Blocks were trimmed so that sections could be cut perpendicular to the pial surface. Silver section ribbons were cut, floated onto 300-mesh grids and stained with uranyl acetate and lead citrate.

\section{Sampling procedure for photography}

Sections selected for study extended from just below the pial surface through 8 - to 9-grid squares (about $765 \mu \mathrm{m}$ ). Two pictures were taken at $\times 11,000$ in each grid square, one in the upper one third and one in the lower one third. Although higher magnification was not used for area selection, obvious neuron and glia somas, capillaries, and major dendrites and axons were avoided during the photography. Only one set of 16 to 18 pictures was made from a section. Prints were made at a final magnification of $\times 24,000$. There were 90 to 120 electron micrographs for each animal.

\section{Quantification}

From the electron micrographs counts were made of ASYM (asymmetrical, distinct postsynaptic opacity) and SYM (symmetrical, equally darkened) membrane appositions. Selection was based upon synaptic membrane configuration and not upon type of vesicles in the presynaptic element.

Counts were made of terminal (bouton) profiles containing round vesicles (RV), flat vesicles (FV) or mixed, RV and FV. Flat and pleomorphic vesicles (Colonnier, '68, '74) tended to be smaller than round vesicles. For purposes here "terminals" were simply profiles containing four or more clearly identifiable vesicles.

Bouton (terminal) areas were measured with a polar planimeter. They were distinguished from "terminals" above by nearly all being selected on the basis of association with either an ASYM or SYM membrane apposition. The bouton to be measured had to be clearly membrane bound so that the extent was not in question. For a given electron micrograph the average bouton area, (a), was related in proportion to the area, (A), of the micrograph, i.e., (a/A). This ratio was used for statistical comparisons.

Since the location of synapses is undoubtedly of functional importance counts were made of synaptic contacts on spines and dendritic shafts. Many elements identified as spines contained a spine apparatus, more or less well defined (fig. 1), or could be seen to be con- nected to a dendritic trunk. The rest were seen as small profiles filled with fluffy material (Peters et al., '76). Dendrites, if not seen in longitudinal section (fig. 1: UC, C), could be identified if transversely cut by the presence of microtubules (Peters et al., '76) (fig. 1: I, C). If a postsynaptic profile could not be positively identified on the basis of these criteria (e.g., fig. 1: UCS, C) it was not counted. For a further comparison the location of synaptic contacts was broken down into two groups, those within about $300 \mu \mathrm{m}$ of the pial surface and those from $300-750 \mu \mathrm{m}$. This would place the structures selected in about layers I and II in the upper sample and in layer III and upper IV in the lower sample (Otsuka and Hassler, '62). Lengths of ASYM and SYM membrane appositions were measured and tabulated. For ASYM appositions the measurement was the length of the clearly identified postsynaptic density. For SYM, measurement was of the length of the region containing equally darkened pre-and postsynaptic membranes, with a wider synaptic cleft and associated slightly dense material. No attempt was made to estimate a total synaptic area. If these data were to be used to establish absolute dimensions, correction for section thickness, and other considerations, would have to be followed, (e.g., Anker and Cragg, '74). Certainly large synaptic contacts were most frequently seen but since all animal tissues were treated equally and section thickness controlled, data comparisons seemed warranted.

Mean differences between groups of measurements were statistically evaluated ( $t$ test, McNemar, '49). It should be noted that since there are only two animals in each of the three groups, a significant difference between means within a group could mean statistical treatment between groups would be inappropriate. For every measure, and in each group, differences between the two animals were statistically evaluated. In the cases where these were significant they will be noted.

\section{RESULTS}

The first electron microscopic work on undercut neocortex (Szentágothai, '65) indicated that both axon-spine and axon-dendritic contacts were considerably reduced. Thinner slabs had greater synaptic loss. The extent of a deviation from normality could only be determined by careful measuring and statistical testing in the present experiments and in 
those of Gruner et al. ('74). Preparations here included more than the six cortical layers, i.e., the undercut was well below cortex. Gliosis, although largely disappearing in gyri isolated for 30 to 46 days (Gruner et al., '74), was still found occasionally within $50 \mu \mathrm{m}$ of the pial surface in undercut cortex in our cats. It was not more apparent in the two cats (UCS) with implanted electrodes. In these animals the presence of the electrodes, dura removal at surgical implant and chronic electrical stimulation apparently did not contribute to increased gliosis. The various synaptic elements, normal in appearance, were readily found in normal and undercut cortex (fig. 1).

For general considerations in this type of electron microscopic quantification it will be helpful to compare some of our data with those of Gruner et al. ('74). They counted in intact suprasylvian gyrus an average of one synapse per $10 \mu \mathrm{m}^{2}$ in the superficial layers. From our sampling we found an average of 5.3 ASYM and SYM membrane appositions per micrograph of $45 \mu \mathrm{m}^{2}$ (I cortex fig. 1: A, B, C), that is, one synapse per $8.5 \mu \mathrm{m}, 18 \%$ more than in the Gruner et al. study. These data may represent real differences between suprasylvian and marginal gyri; they may be the result of differences in tissue fixation or animal preparations; they may result from differences in section thickness; or they may be due to differences in sampling. The synaptic density in isolated suprasylvian gyrus was one synapse per $17 \mu \mathrm{m}^{2}$ (Gruner et al., '74) while in ours it was one per $10 \mu \mathrm{m}^{2}$. Thus, in their study gyrus isolation resulted in a $41 \%$ decrease in synaptic density in superficial cortex; in our undercut cortex synaptic density decreased only $17 \%$. However, in deeper cortical layers they found a decrease of only $20 \%$. On the basis of these findings the superficial layers in undercut marginal gyri contain about $20 \%$ more synapses than do isolated supersylvian gyri.

When tabulating the location of synaptic contacts on dendritic spines and dendritic shafts, Gruner et al. ('74) found in intact superficial layers, $80 \%$ on spines and $20 \%$ on shafts (only axodendritic synapses considered). Our findings for pooled data are $71 \%$ on spines and $29 \%$ on shafts (fig. 5). For their isolated gyri the values were 56 and $44 \%$. For our undercut gyri the values were 54 and $46 \%$ (but see below). From these comparisons it appears the independent studies are in substantial agreement.

\section{Effects of stimulation}

The mean numbers of ASYM and SYM contacts are compared in figure 2 . Undercutting resulted in a $20 \%$ decrease in ASYM contacts. There were no differences between I and UCS. The effects of undercutting alone on SYM contacts seems negligible but actually one UC value was less than the I mean, the other was greater. Chronic stimulation increased SYM contacts, one cat showing $42 \%$ the other $91 \%$ over the I mean. Chronic stimulation seems to have prevented degeneration or loss of ASYM contacts and resulted in more SYM ones being counted. However, since on the average longer contacts are more likely to be seen these findings must be considered with the "length changes" below (fig. 7). This might be a factor in one comparison; ASYM contact length did increase (fig. 7). Therefore, in number of contacts the lack of a difference between UCS and I (fig. 2) conceivably could be explained by the longer contacts of UCS being more frequently seen and counted.

The comparisons of different vesicle containing terminals are shown in figure 3 . Undercutting, with or without stimulation, tended to result in fewer RV terminals. Qualitatively, in the undercut tissue there were great differences in numbers of terminals. Some areas had a large density while others had relatively few terminals. There was no apparent correlation between density of terminals and depth in the superficial layers, presence of glial elements or presence of blood vessels. Undercutting alone did not reliably alter the numbers of terminals containing flat vesi-

Fig. 1 Selected electron micrographs to illustrate some synaptic features of intact (I), undercut (UC) and stimulated undercut (UCS) cortex. Locations of areas were 80-550 $\mu \mathrm{m}$ from the pial surface. Column A shows primarily RA synapses on dendritic spines. Note in I, A, a flat vesicle containing profile which has a poorly differentiated membrane apposition on a dendritic shaft (arrow). This shaft has a stubby spine with a multiple ASYM contact. In UCS, A, one spine has both an RA and an FS synapse, (*). In column B are FS synapses on dendritic shafts (UC, UCS, arrows) and possibly one on a spine (UCS, *). The spine is irregular and has multiple contacts. In $I, B$, the postsynaptic element with a SYM contact from an FV containing profile cannot be identified. Column $\mathrm{C}$ contains a mixture of synaptic loci and types. In $\mathrm{I}, \mathrm{C}$, there is an FS double contact on an trans. versely cut dendritic shaft (arrow); in UC, C, there is a clear FS, with pleomorphic vesicles on a dendritic shaft (arrow) and opposite what may be an RA synapse; and in UCS, C, there are two contacts, at least one likely an FS, on what may be a dendritic shaft (arrow). The plurality of synaptic contacts seen in the UCS row was frequently found in electron micrograph fields not preselected. 

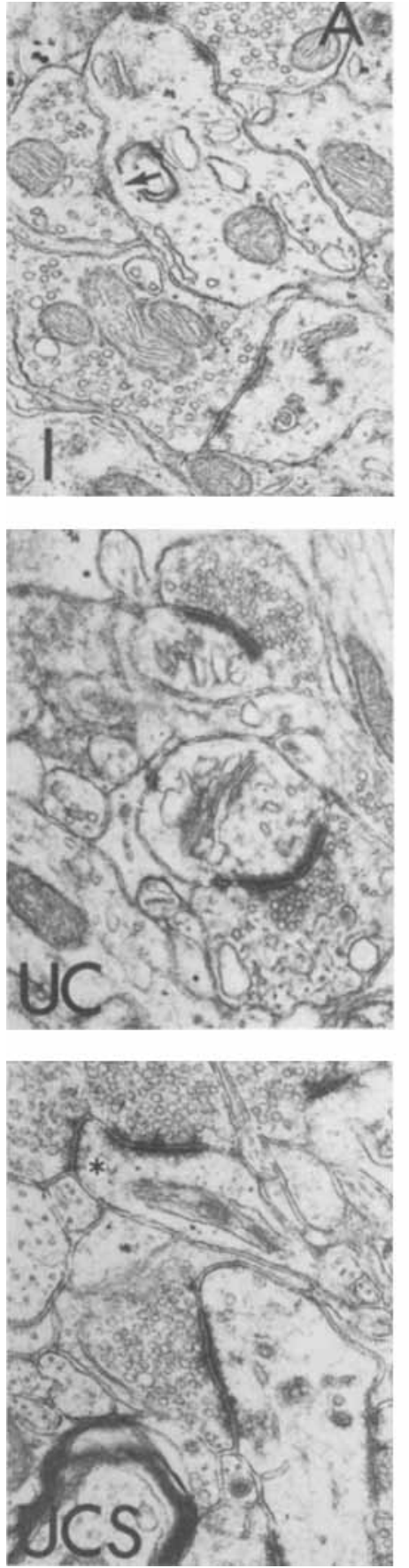
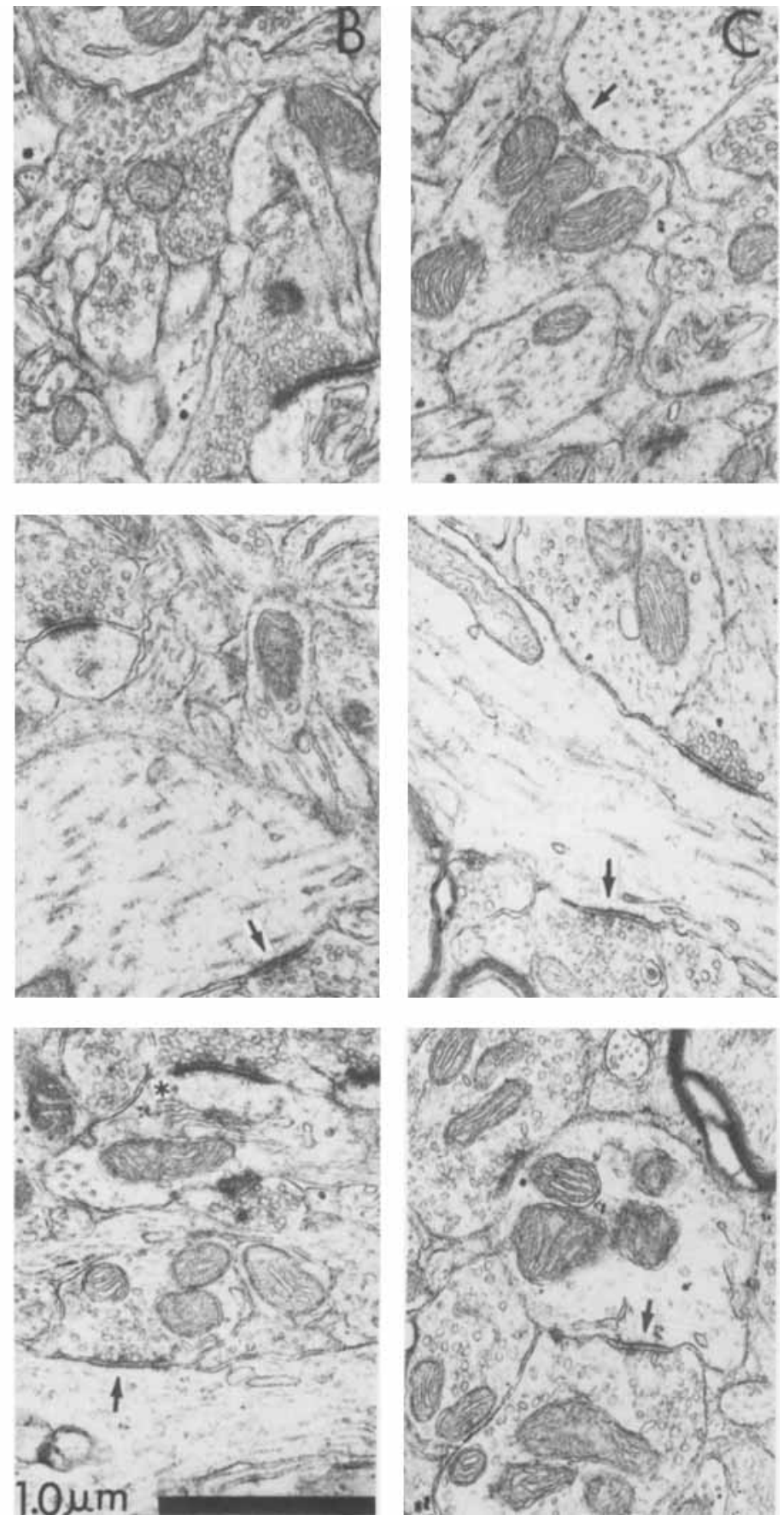

Figure 1 
cles (fig. 3), but stimulation increased the numbers of FV terminals by $69 \%$ and mixed vesicle terminals by $94 \%$.

Different from the "terminal" sampling above where vesicle containing profiles were

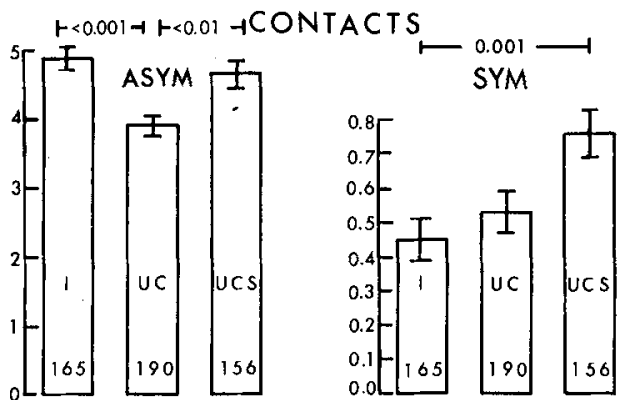

Fig. 2 ASYM and SYM contacts counted in electron micrographs $\left(\times 24,000\right.$, area $\left.45 \mu \mathrm{m}^{2}\right)$. Only contacts with clear membrane differentiations and marked postsynaptic opacity (ASYM) or those with equal pre- and postsynaptic opacities (SYM) were counted. Criteria for selection did not include identification of vesicle population in the presynaptic region nor identification of vesicle population of the postsynaptic structure. Application of these criteria would have greatly reduced the number of contacts to be tabulated. Counts were made from each electron micrograph, the sample sizes being 165 micrographs for intact (I), 190 for undercut (UC) and 156 for stimulated undercut (UCS) cortex. The sample means appear as ordinate values. On the average there was less than one SYM contact in each electron micrograph $\left(45 \mu \mathrm{m}^{2}\right)$. In this and the following figures mean values were compared by student $t$-test and significant differences are indicated by probability levels, other comparisons are not significantly different. The standard error of the mean appears at the top of each bar. counted, areas of those terminals which were membrane bound and usually associated with clearly identified ASYM or SYM contacts were measured and called "boutons." Mean data and comparisons are shown in figure 4. Electrical stimulation resulted in larger bouton areas for both synaptic types. The area increased by $30 \%$ for ASYM and $52 \%$ for SYM. Note that undercutting without stimulation increased the area of boutons associated with SYM contacts by $25 \%$.

Undercutting, with or without stimulation, resulted in an increase in dendritic shaft contacts of $61 \%$ (UC) and $72 \%$ (UCS) (fig. 5). Although spine contacts for UC appear less (18\%) than in $I$, the difference was found largely in only one of the two cases, the decreases being $3 \%$ and $20 \%$. Disregarding this, the $\mathrm{p}$ value for the difference between the pooled area means is 0.01 . Undercutting plus stimulation resulted in significantly more contacts on spines than undercutting without stimulation. Keeping in mind the differences in the two UC animals, (3\% and 30\%), undercutting alone changed the mean proportion of spine and shaft contacts, as compared with intact, from $71 \%$ and $29 \%$ to $54 \%$ and $46 \%$. The decrease for UCS was to $60 \%$ and $40 \%$ (spine and shaft respectively).

Within the superficial layers the effects of denervation and stimulation are not the same when synapse location data from the upper area (layers I and II) are compared with those

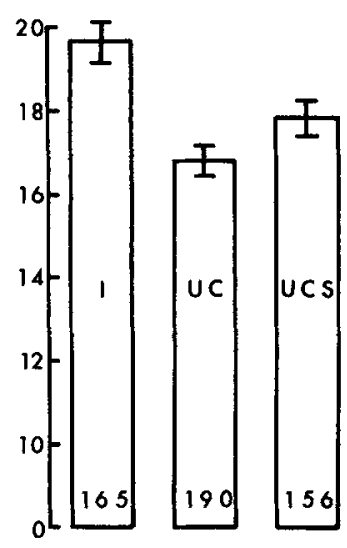

RV
TERMINALS
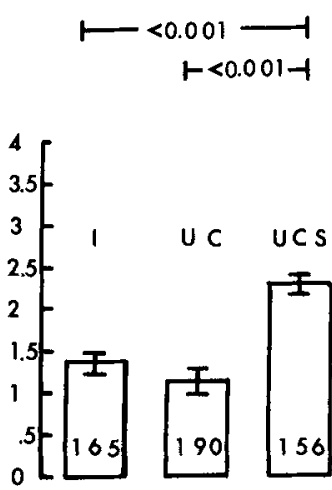

FV
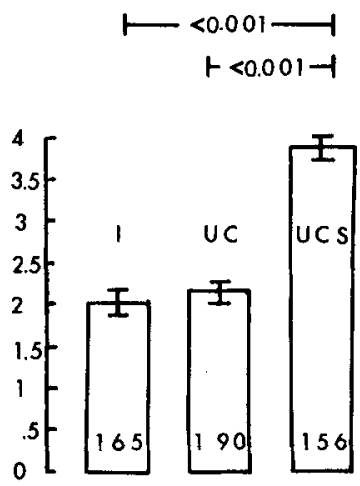

FV (inc. mixed)

Fig. 3 Terminals with different populations of vesicles. Terminals were identified as apparent axon terminals containing four or more clear vesicles. Since the majority could not be associated with a synaptic contact they were distinguished from "boutons." Ordinates are mean values per electron micrograph for sample sizes of 165,190 and 156 . On the average intact cortex (I) had about $20 \mathrm{RV}$ terminals per micrograph $\left(45 \mu \mathrm{m}^{2}\right)$ but only 2 FV terminals (including mixed). Distinctions were made between those containing only round vesicles (RV), only flat vesicles (FV), and those with a mixed population, RV and FV. 


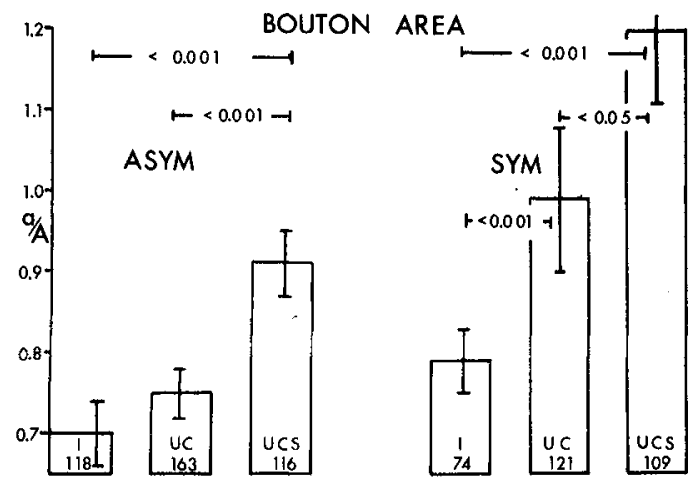

Fig. 4 Mean presynaptic bouton area of RA and FS synapses (a) as a ratio to total area sampled (A). Only those boutons clearly membrane bound were measured. Statistical comparisons were made of the total mean ratio values for the various sample sizes $(118,163$, etc.). For convenience, ratios are $10^{2}$ of actual values.

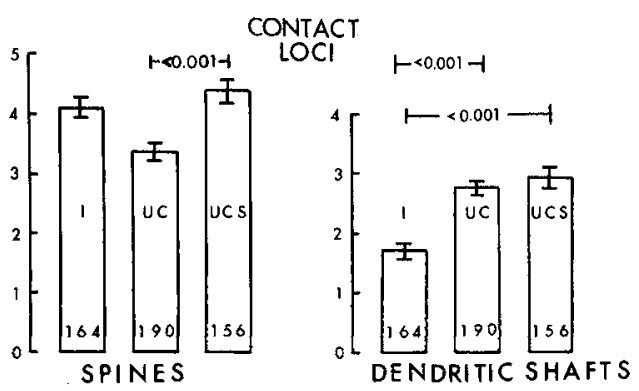

Fig. 5 Location of synaptic contacts. Counts were made in each electron micrograph of the kind of postsynaptic element, whether dendritic spine or dendritic shaft. No distinction was made between ASYM and SYM membrane contacts associated with the postsynaptic structure. Comparisons were of mean values, ordinate, for the sample sizes indicated at the bottom of the bars. from somewhat lower (approximately layer III and upper IV) (fig.6). Although the samples are small for the lower area, and caution in interpretation should be heeded, some interesting and significant differences were found. More spine contacts are lost with undercutting somewhat deeper in the superficial layers, whereas electrical stimulation is "more effective" on spine contacts high in layers I and II. When synaptic locations on dendritic shafts are considered there was a $62 \%$ increase in these contacts at the upper location in undercut cortex. For lower dendritic shaft contacts, within group data variability did not justify a statistical test of I and UC means. There is no similar problem with UCS data. At both locations stimulation resulted in significantly more dendritic shaft contacts (45\% upper, $122 \%$ lower).

Synaptic contact lengths are compared in figure 7. ASYM and SYM contacts average 0.32 and $0.27 \mu \mathrm{m}$ respectively. Undercutting alone did not significantly change these lengths, but with stimulation significant increases of $12 \%$ and $28 \%$ were found.

Table 1 is a summary of the effects of denervation and chronic electrical stimulation on synaptic features in undercut cortex as compared with intact. Denervation alone resulted in synapses associated with symmetric membrane contacts having larger bouton areas and more contacts on dendritic shafts. Chronic stimulation produced increases in, symmetric membrane contacts, areas of round and flat vesicle containing profiles, dendritic shaft contacts and synaptic contact lengths.
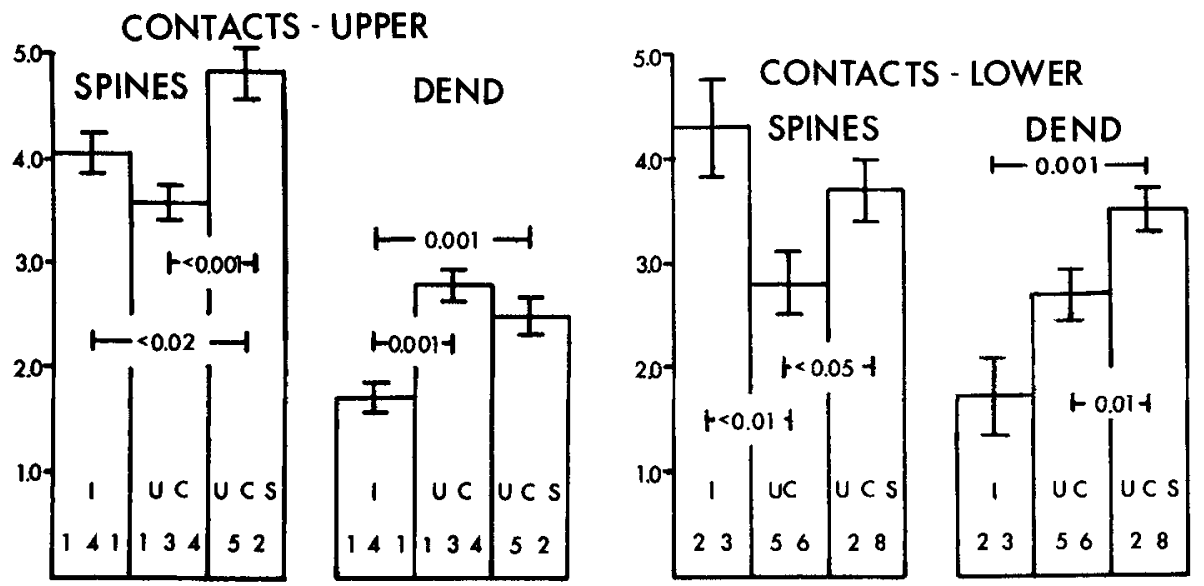

Fig. 6 Mean synaptic contacts per electron micrograph at different depths in cortex. Data were from the "upper" $300 \mu \mathrm{m}$ and "lower," 300-750 $\mu \mathrm{m}$. Sample sizes at bottom of bars. 


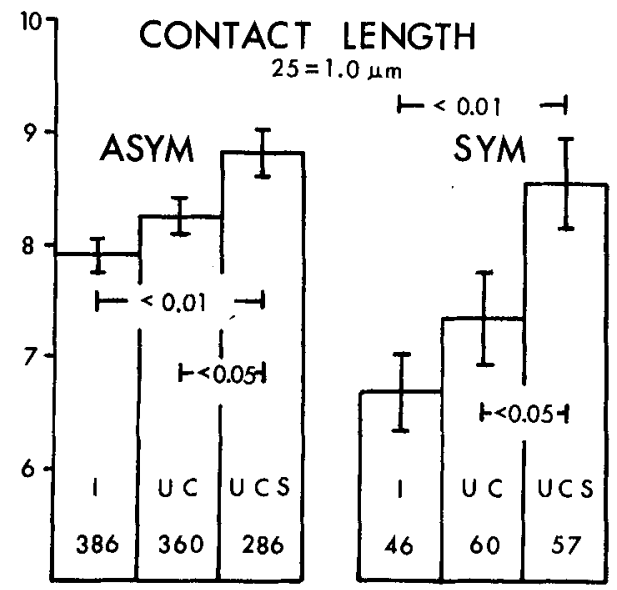

Fig. 7 Comparisons of ASYM and SYM synaptic contact lengths. Where possible vesicle type (RV, FV including mixed) in the presynaptic ending was used to confirm the type of contact. Membrane appositions had to be clearly seen throughout their lengths for selection. The ordinate is on a scale of 25 being equal to $1.0 \mu \mathrm{m}$. Lengths of multiple contacts at a given terminal were added. The number of contact lengths measured in each group is indicated at the bottom of each bar.

TABLE 1

Synaptic features of undercut and stimulated undercut cortex as compared to intact cortex

\begin{tabular}{lcc}
\hline & Undercut & $\begin{array}{c}\text { Stimulated } \\
\text { undercut }\end{array}$ \\
\hline ASYM contacts & - & 0 \\
SYM contacts & NS & + \\
Contact length ASYM & 0 & + \\
Contact length SYM & 0 & + \\
Round vesicle terminals & NS & NS \\
Flat vesicle terminals-all & NS & + \\
Bouton area-RA & 0 & + \\
Bouton area-FS & + & + \\
Contacts on: & & 0 \\
Spines-all & NS & + \\
Dend. shafts-all & + & + \\
Spines-upper & 0 & 0 \\
Spines-lower & - & + \\
Dend. shafts-upper & + & + \\
Dend. shafts-lower & NS & + \\
\hline
\end{tabular}

0, normal; -, decrease; +, increase; RA, round vesicles, ASYM, FS, flat vesicles, symmetrical; NS, not a statistically significant difference.

\section{DISCUSSION}

A problem of concern in the interpretation of these results is the effect of the loss of neurons and attendant collapse or shrinkage of cortical neuropil upon synaptic density. With denervation there would be a synaptic loss and if this was in proportion to neurons lost it could result in no change in synaptic density. In any event shrinkage should be the same in all undercut tissues, assuming undercut tissue thicknesses were about equal, and various measures, purely on a density basis, ought to be the same in UC and UCS. Quantitation in studies using the electron microscope is fraught with many difficulties (Anker and Cragg, '74; Vrensen and De Groot, '73; Weibel, '69). As applied to the CNS one would feel more secure with measurements on ultrastructure if relevant data were available using the light microscope. A basis for undertaking the present work was the previously published quantitative results (Rutledge, '69; Rutledge et al., '69) of studies on dendritic spines and axon collaterals in undercut and stimulated undercut cortex using Golgi-Cox prepared tissues. Those results, as well as those of Benhamida et al. ('70) on dendritic spines in isolated cortex, demonstrated statistically significant losses of these structures. Our present results and those of Gruner et al. ('74) show that with careful sampling, and using simple and constant counting and measuring techniques, reliable quantitative data on synaptic structures following deafferentation and deeferentation of neocortex can be obtained with the electron microscope.

There are two groups of measurements in the present experiments, one associated with ASYM the other with SYM membrane contacts. In neocortex initially fixed with aldehyde, certain elements of synapses are most frequently found together (Colonnier, '68). Usually ASYM contacts have distinct postsynaptic opacities and presynaptic structures containing round vesicles while SYM contacts have membrane appositions equally darkened and a presynaptic terminal with small, flat vesicles. As pointed out by Colonnier ('68, '74) these types correspond closely to but are not strictly the same as Gray's Types 1 and 2 (Gray, '59). For convenience here, the terms RA (round vesicles, asymmetrical membrane appositions) synapses and FS (flat vesicles, symmetrical membrane appositions) synapses will be used to distinguish the two types.

Denervation by undercutting, and without stimulation, resulted in a significant loss of ASYM contacts (table 1) but other measures of RA synaptic elements were variable, especially the counts of RV terminals in both UC and UCS. The identification of terminals with 
vesicles was difficult. It could not be assumed that every profile containing vesicles was a part of a synaptic complex since a clustering of four or more vesicles could appear in profiles that were not terminal, notably within the axon itself. Further, there were area differences with the overall density in some electron micrographs being greater than in others. Of course this pertains to both RV and FV terminals, but in the case of the latter to find four FV alone was unusual and positive identification for FV frequently resulted in counting only those terminals where many vesicles had a marked "flatness."

Light microscopic work found significant losses in dendritic spines in undercut cortex (Rutledge, '69) or in isolated cortex (Benhamida et al., '70). Further, qualitative studies showed similar effects of cortical denervation (Cajal, '59; Szentágothai, '65; Westrum et al., ' 64 ). In the present study only one location, 300-750 $\mu \mathrm{m}$ below the cortical surface, had a significant loss of contacts on spines with undercutting, but this measurement is not the same as counting spines in light microscopy. Also, with the Golgi-Cox technique and using the light microscope the spines most easily counted and noticed when not present are the largest ones and in our previous study (Rutledge, '69) these were on upper layer pyramidal cells. With electron microscopy all spine contacts large and small were counted, the parent structure unidentified. Data from the two studies are not necessarily comparable. For round asymmetrical synapses the effects of electrical stimulation are clear, except for the variability in numbers of RV terminals (table 1). Stimulation increased contact length, bouton area and spine contacts in the most upper part of the cortex. These effects are more profound than those found with the light microscope in UCS (Rutledge, '69; Rutledge et al., '69), since no increases were observed in any of these structures measured in Golgi-Cox material. Increases in neuronal structures were seen in another type of experiment wherein direct cortical stimulation was the causal factor (Rutledge et al., '74).

Except for an increase in bouton area undercutting alone had a variable effect upon elements of FS synapses. This is in contrast to the effects of stimulation. In all four measures, numbers of SYM contacts, FV terminals, bouton area and contact length, there were significant increases over normal cortical values (table 1). Thus stimulation had a decidedly stronger effect upon FS synapses than upon RA synapses.

A provocative observation in the present study, as compared with light microscopic work in which the interpretation was that stimulation prevented spine loss from an expected $40 \%$ (UC) to only $7 \%$ (UCS), is that with the exception of RV terminals, stimulation resulted in normal numbers of synaptic elements or increased the numbers and sizes. Chronic stimulation cannot preserve afferent axons (terminals) and synaptic elements and therefore the alterations in fine structure as a result of stimulation must be thought of as resulting from changes at synapses served by intracortical axons-as compared with normal cortex. Increases in synaptic dimensions, numbers, etc. would be interpretable as growth changes of remaining synaptic elements. Growth changes could involve sprouting of terminals onto existing spines or onto newly formed spines. There is evidence at least for the latter in our earlier light microscopic data (Rutledge, '69; Rutledge et al., '69). If the earlier data are valid measures of spine populations, and there is a $40 \%$ decrease in spines in denervated cortex but only $7 \%$ in stimulated cortex, then stimulation must increase numbers of spines served by intracortical terminals rather than preserve spines denuded of endings from afferent axons. To what extent vacated spines may be reoccupied by intracortical terminals is unknown.

In neocortex most RA synapses are associated with dendritic spines and most FS synapses are on dendritic shafts (Colonnier, '68). There are exceptions, and there are mixtures of elements, but if these are disregarded, undercutting alone increased FS synapses in the most upper part of cortex and decreased RA synapses in the lower portion. There was thus a differential effect, suggesting, by the presence of the specifically located new shaft contacts, a compensatory response to denervation, perhaps axonal sprouting. Although details are lacking, there is a recent mention of an increase in Gray's Type 2 synapses in isolated cortex (Ulmar et al., '76). Both spine and dendritic shaft contacts are increased, or no different from intact cortex, in UCS. Stimulation apparently promoted growth of RA and FS synaptic complexes.

There are functional implications of these 
observations if it is assumed that RA synapses are equivalent to Gray's Type 1 synapses, FS synapses are equivalent to Gray's Type 2 and the two types function, respectively, as excitatory and inhibitory structures (Larramendi et al., '67; Lund and Westrum, '66; Uchizono, '68; Walberg, '68). Inhibition is still operative in undercut cortex (Krnjevic et al., '70a,b) but there is actually a $10-25 \%$ loss (Anderson et al.,'75). It is suggested that there is an aborted attempt by inhibitory neu. rons to maintain functional integrity in denervated cortex. Bouton area and dendritic shaft contacts in upper cortex increased in FS synapses. If new shaft contacts form, axon terminal sprouting seems the most likely explanation. Swelling of terminal boutons would be a more subtle expression of axonal growth. There is no direct evidence from the present experiments that would explain "supersensitivity" in undercut cortex. However, the effects of stimulation on fine-structure and our previous work showing that "supersensitivity" is not present in UCS, indicates that stimulation produces new functional inhibition. It is suggested that this result is mediated by the growth of new FS synapses in UCS. Further, normal excitatory circuits are maintained as indicated by the fine-structure of RA synapses (with the exception of RV terminals).

The changes observed in UCS can be best described as synaptic growth or remodeling. We feel that chronic electrical stimulation produced neuronal activity and increased synaptic use resulting in the described effects. There are other data which point to growth plasticity following denervation in adult, mature nervous systems, for examples, in septal nuclei (Raisman and Field, '73), in adrenergic neurons (Moore et al., '74; Stenevi et al., '73), in the hippocampus (Lynch et al., '73; Matthews et al., '76; Steward et al., '74, '76) and in VPL of the thalamus (Wall and Egger, '71). Still other experiments indicate that plasticity of structure can be produced or accelerated by specific stimulation. Fehér et al. ('72) showed that in adult cats 30 to 45 minutes of acoustic stimulation produced either an increase or a decrease in vesicle density in axon terminals in layers IV and V of cat cerebral cortex. Following a short period of stimulation of the perforant path, spines in the fascia dentata were swollen, indicative of increased syn. aptic activity (Van Harreveld and Fifkova,
'75). Synapses in layer III of the cerebral cortex were larger as a result of supposedly increased synaptic activity in rats subjected to an enriched environment (Møllgaard et al., '71), and exposure to a complex environment produced an increase in dendritic branching of neurons in occipital cortex (Greenough and Volkmar, '73). There are numerous reports showing that neuronal structure and function are especially plastic in the developing or immature brain. For example, in a recent quantitative electron microscope study of kitten neocortex which had received sensory input for the first time at one month of age, Garey and Pettigrew ('74) found a significant increase in axon terminals and vesicle density.

It now seems certain that many neurons of the central nervous system can express considerable plasticity following lesions, even in the adult brain. Partial denervation of an area can provide a convenient and useful method of revealing synaptic growth processes and as emphasized in the present work, synaptic and functional remodeling can be promoted by electrical stimulation of denervated tissue containing complex neuronal circuitry.

\section{ACKNOWLEDGMENTS}

This work was supported by N. I. H. Research Grant NS04119. I am grateful to Doctor Marc Colonnier for guidance in the initial phases of this research and to Mrs. Cheryl Wright for fine technical assistance.

\section{LITERATURE CITED}

Anderson, T. E., L. T. Rutledge and R. S. Dyer 1975 Altered neuronal excitability accompanying experimental prevention of supersensitivity in undercut cortex. Brain Res., 95: 147-153.

Anker, R. L., and B. G. Cragg 1974 Estimation of the number of synapses in a volume of nervous tissue from counts in thin sections by electron microscopy. J. Neurocytol., 3: 725-735.

Benhamida, C., G. Ruiz de Pereda and J. C. Hirsch 1970 Les épines dendritiques du cortex de gyrus isolé de chat. Brain Res., 21: 313.325.

Cajal, S. R. y. 1959 Degeneration and Regeneration of the Nervous System. Vol. 2, R. M. May (translator), reprinted by Hafner, New York.

Chu, N.-S., L. T. Rutledge and O. Z. Sellinger 1971a The effect of cortical undercutting and long-term electrical stimulation on synaptic acetylcholinesterase. Brain Res., 29: 323.330 .

$1971 \mathrm{~b}$ Tubocurarine binding in undercut cerebral cortex and the effect of long-term electrical stimulation. Brain Res., 29: 331-337.

Colonnier, M. 1968 Synaptic patterns on different cell types in the different laminae of the cat visual cortex. An electron microscope study. Brain Res., 9: 268-287. 
1974 Spatial interrelationships as physiological mechanisms in the central nervous system. In: Essays on the Nervoue System. R. Bellairs and E. G. Gray, eds. Clarendon Press, Oxford, England, pp. 344-366.

Cooper, I. S., M. Riklan, I. Amin, J. M. Waltz and T. Cullinan 1976 Chronic cerebellar stimulation in cereberal palsy. Neurology, 26: 744-753.

Dobelle, W. H., M. G. Mladejovsky, J. R. Evans, T. S. Roberts and J. P. Girvin 1976 "Braille" reading by a blind volunteer by visual cortex stimulation. Nature, 259: 111-112

Duncan, J. A., L. T. Rutledge and E. F. Domino 1968 Acetylcholinesterase activity in partially isolated cerebral cortex after prolonged intermittent stimulation. Exp. Neurol., 20: 268-274.

Fehér, O., F. Joó and N. Halász 1972 Effects of stimulation on the number of synaptic vesicles in nerve fibres and ter. minals of the cerebral cortex in the cat. Brain Res., 47 37.48.

Fields, H. L., and J. E. Adams 1974 Pain after cortical injury relieved by electrical stimulation of the internal capsule. Brain, 97: 169-178

Garey, L. J., and J. D. Pettigrew 1974 Ultrastructural changes in kitten visual cortex after environmental mod ification. Brain Res,, 66: 165-172.

Gray, E. G. 1959 Axo-somatic and axo-dendritic syn apses of the cerebral cortex: An electron microscope study. J. Anat., 93: 420-433.

Greenough, W. T., and F. R. Volkmar 1973 Pattern of dendritic branching in occipital cortex of rats reared in com plex environments. Exp. Neurol., 40: 491-504.

Gruner, J. E., J. C. Hirsch and C. Sotelo 1974 Ultrastructural features of the isolated suprasyvvan gyrus in the cat. J. Comp. Neur., 154: 1-28.

Krnjević, K., R. J. Reiffenstein and A. Silver 1970a Chemical sensitivity of neurons in long isolated slabs of cat cerebral cortex. Electroenceph. Clin. Neurophysiol., 29: 269-282.

$1970 \mathrm{~b}$ Inhibition and paroxysmal activity in long isolated cortical slabs. Electroenceph. Clin. Neurophysiol., 29: 283-294.

Larramendi, L. M. H., L. Rickenscher and N. Lemkey-Johnston 1967 Synaptic vesicles of inhibitory and excitatory terminals in the cerebellum. Science, 156: 967-969.

Lund, R. D., and J. S. Lund 1971 Synaptic adjustment after deafferentation of the superior colliculus of the rat. Sci ence, 171: 804.804 .

Lund, R. D, and L. E. Westrum 1966 Synaptic vesicle differences after primary formalin fixation. J. Physiol. (London), 185: 7.9 .

Lynch, G. S., S. Mosko, T. Parks and C. W. Cotman 1973 Relocation and hyperdevelopment of the dentate gyrus commissural system after entorhinal lesions in immature rats. Brain Res., 50 : 174.178 .

McNemar, Q. 1949 Psychological Statistics. John Wiley and Sons, Inc., New York.

Matthews, D. A., C. Cotman and G. Lynch 1976 An electron microscopic study of lesion-induced synaptogenesis in the dentate gyrus of the adult rat. II. Reappearance of morphologically normal synaptic contacts. Brain Res., 115: 23-41.

Mollgaard, K., M. C. Diamond, E. L Bennett, M. R. Rosenzweig and B. Lindner 1971 Quantitative synaptic changes with differential experience in rat brain. Int. $J$. Neurosci., 2: 113-128.

Moore, R. Y., A. Björklund and U. Stenevi 1974 Growth and plasticity of adrenergic neurons. In: The Neurosciences: Third Study Program. F. O. Schmitt and F. G. Worden, eds. M. I. T. Press, Cambridge, Massachusetts, pp. 961-977.
Otsuka, R., and R. Hassler 1962 Über aufbau und gliederung der corticalen sehsphäre bei der katze. Neurologie, 203: 212-234.

Peters, A., S. L. Palay and H. De F. Webster 1976 The Fine Structure of the Nervous System. W. B. Saunders, Philadelphia.

Raisman, G., and P. M. Field 1973 A quantitative investigation of the development of collateral reinnervation after partial deafferentation of the septal nuclei. Brain Res., 50: 241-264.

Richardson, D. E. 1976 Brain stimulation for pain control. IEEE Trans. Biomed. Eng., 23: 304-306.

Rutledge, L. T. 1969 Effect of stimulation on isolated cortex. In: Basic Mechanisms of the Epilepsies. Little, Brown and Company Inc., Boston, Massachusetts, pp. 349-355.

1975 Presumed inhibitory synapses produced by electrical stimulation of undercut neocortex. In: Neuroscience Abstracts. Vol. 1, Society for Neuroscience, Bethesda, Maryland, p. 711.

Rutledge, L. T., J. A. Duncan and N. Beatty 1969 A study of pyramidal cell axon collaterals in intact and partially isolated adult cerebral cortex. Brain Res., 16: 15-22.

Rutledge, L. T., J. B. Ranck, Jr. and J. A. Duncan 1967 Prevention of supersensitivity in partially isolated cerebral cortex. Electroenceph. Clin. Neurophysiol., 23: 256-262.

Rutledge, L. T., C. Wright and J. Duncan 1974 Morphological changes in pyramidal cells of mammalian neocortex associated with increased use. Exp. Neurol, 44: 209-228.

Sharpless, S., and L. Halpern 1962 The electrical excitability of chronically isolated cortex studied by means of permanently implanted electrodes. Electroenceph. Clin. Neurophysiol., 14: 224-255.

Stenevi, U., A. Björklund and R. Y. Moore 1973 Morphological plasticity of central adrenergic neurons. Brain, Behav, and Evol., 8: 110-134.

Steward, O., C. W. Cotman and G. S. Lynch 1974 Growth of a new fiber projection in the brain of adult rats: Re-innervation of the dentate gyrus by the contralateral entorhinal cortex following ipsilateral entorhinal lesions. Exp. Brain Res., 20: 45-66.

1976 A quantitative autoradiographic and electrophysiological study of the reinnervation of the dentate gyrus by the contralateral entorhinal cortex following ipsilateral entorhinal lesions. Brain Res., 114: 181-200.

Szentágothai, J. 1965 The synapses of short local neurons in the cerebral cortex. Symp. Biol. Hung., 5: 251-276.

Uchizono, K. 1968 Inhibitory and excitatory synapses in vertebrate and invertebrate animals. In: Structure and Function of Inhibitory Neuronal Mechanisms. C. von Euler, S. Skoglund and U. Söderberg, eds. Pergamon Press, New York, pp. 33-58.

Ulmar, G., G. Vickers, M. J. Dowdall and V. Neuhoff 1976 Pre-fractionation of rat cortical nerve endings by experimental deafferentation. Exp. Brain Res., 24: 22.

Van Haareveld, A., and E. Fifkova 1975 Swelling of dendritic spines in the fascia dentata after stimulation of the perforant fibers as a mechanism of post-tetanic potentation. Exp. Neurol., 49: 736-749.

Vrensen, G., and D. De Groot 1973 Quantitative stereology of synapses: A critical investigation. Brain Res., 58: 25-35.

Walberg, F. 1968 Morphological correlates of postsynaptic inhibitory processes. In: Structure and Function of Inhibitory Neuronal Mechanisms. C. von Euler, S. Skoglund and U. Söderberg, eds. Pergamon Press, New York, pp. 7-12.

Wall, P. D., and M. D. Egger 1971 Formation of new con- 
nexions in adult rat brains after partial deafferentation Nature, 232: 542-545.

Weibel, E. R. 1969 Stereological principles for morphom. etry in electron microscopic cytology. Int. Rev. Cytol., 26 . 235-302.

Westrum, L. E., and R. G. Black 1971 Fine structural aspects of the synaptic organization of the spinal trigeminal nucleus (pars interpolaris) of the cat. Brain Res., 25: 265-283.

Westrum, L. E., L. E. White, Jr. and A. A. Ward, Jr. 1964 Morphology of the experimental epileptic focus. J. Neurosurg., 21: 1033-1046. 